\title{
COMMENT
}

Check for updates

\section{Cardiovascular health and disease in migrant populations: a call to action}

Charles Agyemang $\mathbb{1}^{1 凶}$ and Bert-Jan van den Born ${ }^{1,2}$

Large differences in cardiovascular disease (CVD) morbidity and mortality exist between migrant populations and host populations. Understanding the drivers behind these disparities may help to mitigate the unequal burden of CVD and identify new causal pathways that contribute to CVD risk in the population at large.

migration
generally has
an adverse
effect on health,
particularly
cardiovascular
and metabolic
health

'Department of Public \& Occupational Health, Amsterdam Public Health Research Institute, Amsterdam UMC, University of Amsterdam, Amsterdam, Netherlands.

${ }^{2}$ Department of Vascular Medicine, Amsterdam Cardiovascular Sciences, Amsterdam UMC, University of Amsterdam, Amsterdam, Netherlands.

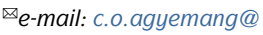
amsterdamumc.nI

https://doi.org/10.1038/ s41569-021-00644-y
Human migration is profoundly reshaping the world population, especially in urban centres in high-income countries in Europe and North America. Migration is a double-edged sword. On the one hand, migration can provide social and economic benefits through improved access to education and an increase in income, as well as a safety net for those fleeing violence and persecution. However, migration generally has an adverse effect on health, particularly cardiovascular and metabolic health, which is more pronounced among migrants moving to high-income countries ${ }^{1}$. Furthermore, relocation from rural to urban regions is also associated with elevated risk of cardiovascular disease (CVD).

Despite the overall improvement in CVD outcomes in the past decade among the general population living in high-income countries in Europe and North America, large differences in CVD morbidity and mortality exist between migrants and the host populations in these countries $^{1}$. These differences are attributable to numerous factors, including migrant background, country of residence and length of stay (FIG. 1). In many instances, migrants have worse CVD outcomes compared with the host population, but in some settings the migrant population seems to have better outcomes than the host population. For example, in Spain, CVD mortality was found to be higher in migrants from Asia, the Caribbean and sub-Saharan Africa than in the Spanish host population, but was lower in those born in North Africa and South America ${ }^{2}$. Likewise, in the Netherlands, overall CVD mortality was higher among male migrants from Suriname compared with the Dutch general population, but was $50 \%$ lower among male migrants from Morocco $^{3}$. These data become more complex when taking into account specific CVD outcomes such as stroke and coronary heart disease. A systematic review showed that migrants living in Western Europe, especially those from South Asia, the Middle East and Eastern Europe, had a similar or higher risk of ischaemic heart disease and stroke than the host population, whereas migrants from North Africa had a lower risk of stroke ${ }^{4}$. A New York study that reported the rates of stroke subtypes in the population revealed a lower rate of ischaemic stroke in Chinese individuals compared with white individuals, but a higher mortality from haemorrhagic stroke ${ }^{5}$. Notably, the context of the host country also contributes to differences in CVD risk. For example, Chinese migrants in the Netherlands have a lower risk of stroke than the Dutch general population, whereas Chinese migrants in Sweden have a higher risk of stroke than the host Swedish population ${ }^{4}$. These country-specific differences might be owing, in part, to the migrant pond effect (that is, the health status of the host population in the country of settlement is crucial for the health of migrants, given that they are the reference populations to which migrant populations are compared) or differential exposure to country-specific contextual risk factors.

Global studies on the relationship between risk factors and CVD, such as INTERHEART and INTERSTROKE, have shown that the effect of a risk factor on ischaemic heart disease and stroke is dependent not only on disease prevalence, but also on the coexistence of and interaction with other CVD risk factors ${ }^{6}$. The higher prevalence of obesity, diabetes mellitus and hypertension in most migrant groups compared with the host population might, therefore, explain a large part of the observed discrepancies in CVD outcomes. However, within the same geographical context, large differences in the prevalence of these risk factors also exist between migrant groups. For example, the prevalence of type 2 diabetes is higher in all migrant groups than in the European host populations, with the highest prevalence observed in South Asian migrants, whereas the prevalence of hypertension is highest in migrants of West African descent ${ }^{1}$.

The differences in CVD prevalence and risk factors between the migrant population and the host population have been attributed to factors such as migration-related lifestyle changes, psychosocial stress and low socioeconomic status, as well as discrepancies in genetic susceptibility and gene-environment interactions ${ }^{1}$ (FIG. 1). At present, very few genetic variants have been identified that, as a result of natural selection, directly influence CVD risk. However, analyses of several common 


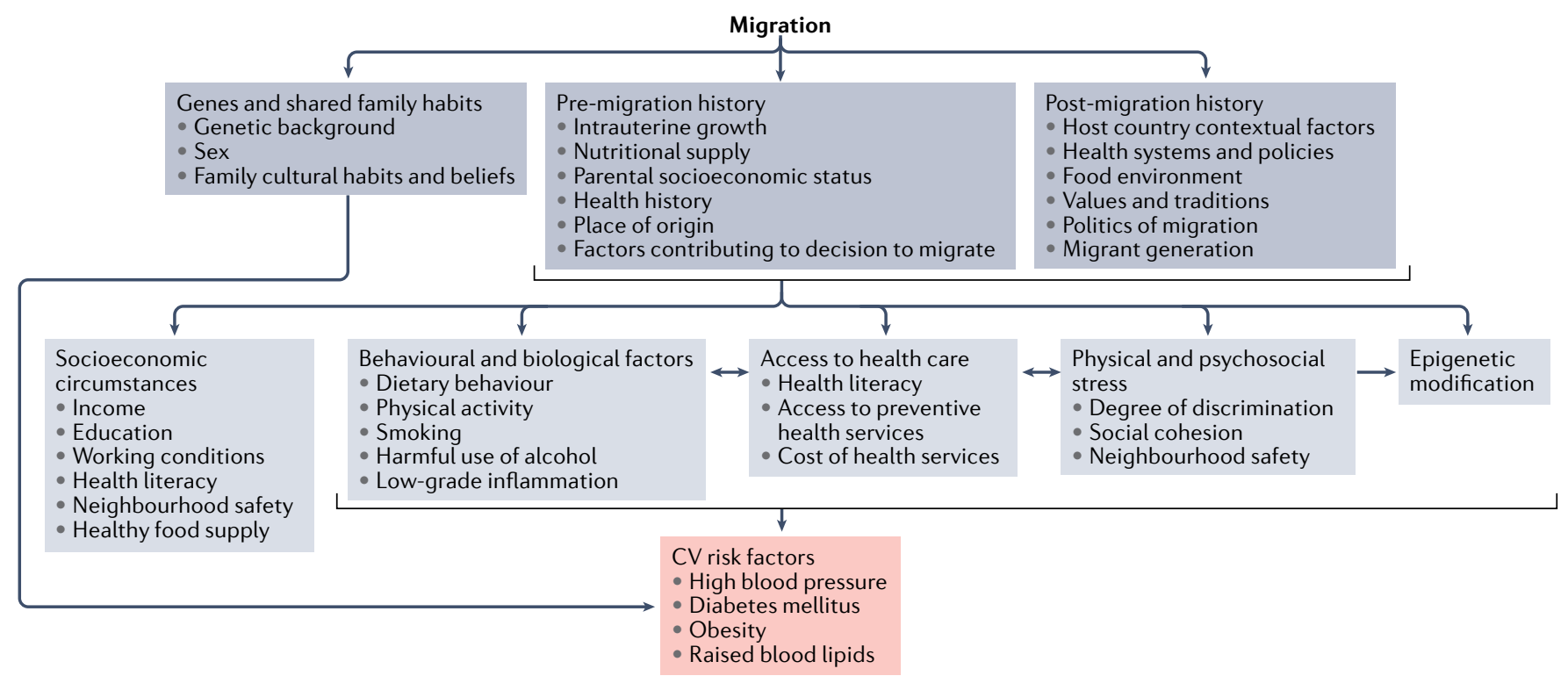

Fig. 1 | Factors contributing to cardiovascular disease in migrant populations. Numerous variables can contribute to the risk of cardiovascular (CV) disease among migrants, including those related to genetics, place of origin, socioeconomic status, physical and psychosocial stress and access to health care.

polymorphisms associated with salt sensitivity and heat stress suggest that polygenic traits might influence the propensity to develop risk factors, such as hypertension and diabetes ${ }^{7}$. Additionally, epigenetic alterations might also influence the risk of cardiometabolic diseases among transitioning populations, as evidenced by differential DNA methylation sites that affect biological ageing . Differences in CVD risk between different migrant groups and between the migrant population and the host population might also be influenced by metagenomic traits, in particular the gut microbiome. Variations in the diversity and composition of the gut microbiota between migrants and the host population has been observed'. Faecal microbiota composition has also been linked with blood pressure levels, but with strongly divergent associations across different ethnic groups ${ }^{10}$.

Regardless of their cause, the higher rates of cardiovascular risk factors among migrants do not bode well for their future. Although the high risk of CVD among migrants has been known for several decades, the key underlying drivers of this increased risk are still not well understood. A better understanding of the causes of the disparities in CVD risk will not only help to mitigate the unequal burden of CVD, but also facilitate the identification of new causal pathways that are relevant to CVD risk for the population at large. At present, initiatives to fund research aimed at exploring the disparities in CVD risk among multi-ethnic populations are lagging behind. Although the health challenges facing migrants and the need to address them have been well acknowledged, financial sponsorship from governments and funding institutions has been meagre and has not kept pace with the extent of the challenges. In the Netherlands, for example, cohort studies that are aimed at exploring differences between multi-ethnic populations have received much less funding than other cohort studies, because funding depends on location in economical regions of interest rather than disparities in health. Nevertheless, the health disparities among migrant populations are very costly in terms of both economics and human suffering. The disproportionate burden of the coronavirus disease 2019 (COVID-19) pandemic on the migrant population is driven, in part, by the higher prevalence of pre-existing chronic conditions, including CVD and its risk factors, in this population compared to native-born populations, as well as the higher incidence of poverty and the greater likelihood of having a job in which physical distancing is difficult. These observations should not only serve as a wake-up call that these disparities in CVD risk exist, but should also prompt action to understand and mitigate these differences.

1. Agyemang, C. $\&$ van den Born, B.-J. Non-communicable diseases in migrants: an expert review. J. Travel Med. 26, tay 107 (2019).

2. Regidor, E. et al. Mortalidad por enfermedades cardiovasculares en inmigrantes residentes en la Comunidad de Madrid. Med. Clin. (Barc.) 132, 621-624 (2009)

3. Bos, V., Kunst, A. E., Keij-Deerenberg, I. M., Garssen, J. \& Mackenbach, J. P. Ethnic inequalities in age- and cause-specific mortality in The Netherlands. Int. J. Epidemiol. 33, 1112-1119 (2004).

4. Sohail, Q. Z. et al. The risk of ischemic heart disease and stroke among immigrant populations: a systematic review. Can. J. Cardiol. 31, 1160-1168 (2015)

5. Fang, J., Madhavan, S. ¿ Alderman, M. H. Cardiovascular mortality of Chinese in New York City. J. Urban Health 76, 51-61 (1999).

6. Yusuf, S. et al. Effect of potentially modifiable risk factors associated with myocardial infarction in 52 countries (the INTERHEART study): case-control study. Lancet 364, 937-952 (2004).

7. Adeyemo, A. A., Shriner, D., Bentley, A. R., Gbadegesin, R. A $\&$ Rotimi, C. N. Evolutionary genetics and acclimatization in nephrology. Nat. Rev. Nephrol. https://doi.org/10.1038/ s41581-021-00483-7 (2021).

8. Chilunga, F. P. et al. Epigenetic-age acceleration in the emerging burden of cardiometabolic diseases among migrant and nonmigrant African populations: a population-based cross-sectional RODAM substudy. Lancet Healthy Longev. 2, E327-E339 (2021).

9. Deschasaux, M. et al. Depicting the composition of gut microbiota in a population with varied ethnic origins but shared geography. Nat. Med. 24, 1526-1531 (2018)

10. Verhaar, B. J. H. et al. Associations between gut microbiota, faecal short-chain fatty acids, and blood pressure across ethnic groups: the HELIUS study. Eur. Heart J. 41, 4259-4267 (2020).

\section{Acknowledgements}

C.A. is supported by the European Research Council (consolidator grant \#772244).

\section{Competing interests}

The authors declare no competing interests. 